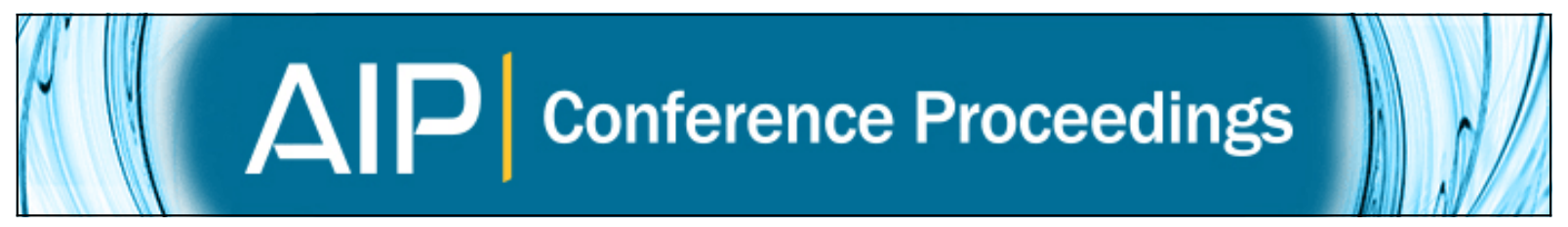

\title{
The MKID Camera
}

P. R. Maloney, N. G. Czakon, P. K. Day, R. Duan, J. Gao, J. Glenn, S. Golwala, M. Hollister, H .G. LeDuc, B. Mazin, O. Noroozian, H. T. Nguyen, J. Sayers, J. Schlaerth, J. E. Vaillancourt, A. Vayonakis, P. Wilson, and J. Zmuidzinas

Citation: AIP Conference Proceedings 1185, 176 (2009); doi: 10.1063/1.3292309

View online: http://dx.doi.org/10.1063/1.3292309

View Table of Contents: http://scitation.aip.org/content/aip/proceeding/aipcp/1185?ver=pdfcov

Published by the AIP Publishing

\section{Articles you may be interested in}

Future Developments in Low Temperature Detectors for CMB and Submm Astronomy

AIP Conf. Proc. 1185, 773 (2009); 10.1063/1.3292454

SPT-SZ: a Sunyaev-ZePdovich survey for galaxy clusters

AIP Conf. Proc. 1185, 475 (2009); 10.1063/1.3292381

Optical Properties of Argonne/KICP TES Bolometers for CMB Polarimetry

AIP Conf. Proc. 1185, 203 (2009); 10.1063/1.3292315

Sensitivity Optimization of Millimeter/Submillimeter MKID Camera Pixel Device Design

AIP Conf. Proc. 1185, 180 (2009); 10.1063/1.3292310

Microwave Kinetic Inductance Detector (MKID) Camera Testing for Submillimeter Astronomy

AIP Conf. Proc. 1185, 172 (2009); 10.1063/1.3292308 


\title{
The MKID Camera
}

\author{
P.R. Maloney*, N.G. Czakon ${ }^{\dagger}$, P.K. Day**, R. Duan ${ }^{\dagger}$, J. Gao ${ }^{\ddagger}$, J. Glenn*, S. \\ Golwala $^{\dagger}$, M. Hollister ${ }^{\dagger}$, H.G. LeDuc**, B. Mazin ${ }^{\S}$, O. Noroozian ${ }^{\dagger}$, H.T. Nguyen ${ }^{* *}$, \\ J. Sayers ${ }^{* *}$, J. Schlaerth*, J.E. Vaillancourt ${ }^{\dagger}$, A. Vayonakis ${ }^{\dagger}$, P. Wilson ${ }^{* *}$ and J. \\ Zmuidzinas $^{\dagger}$ \\ ${ }^{*}$ CASA, University of Colorado, Boulder CO 80303 \\ ${ }^{\dagger}$ California Institute of Technology, Pasadena, CA 91125 \\ ** Jet Propulsion Laboratory, Pasadena, CA, 91109 \\ $\$$ National Institute of Standards and Technology, Boulder CO 80305 \\ $\S$ University of California, Santa Barbara, CA, 93106
}

\begin{abstract}
The MKID Camera project is a collaborative effort of Caltech, JPL, the University of Colorado, and UC Santa Barbara to develop a large-format, multi-color millimeter and submillimeter-wavelength camera for astronomy using microwave kinetic inductance detectors (MKIDs). These are superconducting, micro-resonators fabricated from thin aluminum and niobium films. We couple the MKIDs to multi-slot antennas and measure the change in surface impedance produced by photon-induced breaking of Cooper pairs. The readout is almost entirely at room temperature and can be highly multiplexed; in principle hundreds or even thousands of resonators could be read out on a single feedline.

The camera will have 576 spatial pixels that image simultaneously in four bands at $750,850,1100$ and 1300 microns. It is scheduled for deployment at the Caltech Submillimeter Observatory in the summer of 2010 . We present an overview of the camera design and readout and describe the current status of testing and fabrication.
\end{abstract}

Keywords: Radio Telescopes and Instrumentation. Superconducting infrared, submillimeter, and millimeter-wave detectors PACS: $95.55 . \mathrm{Jz}, 85.25 . \mathrm{Pb}$

\section{INTRODUCTION}

The development of large-format arrays of incoherent detectors for sub/millimeter astronomy has proven to be technologically challenging, especially due to the difficulties in reading out thousands of detectors simultaneously. Considerable progress has been made recently using a novel type of device, microwave kinetic inductors, or MKIDS. We are developing a 576-pixel, 4-color astronomical instrument, the MKID camera, which will be deployed at the CSO on Mauna Kea in 2010.

\section{MKIDS}

The physics of MKIDs and their applications are discussed in detail by [2], and references therein; here we present only the directly relevant aspects.

Superconductors have a non-zero impedance for ac currents, as the paired-up electrons which carry the supercurrent, known as Cooper pairs, can be accelerated and decelerated by an electric field, allowing energy to be stored in (or extracted from) the kinetic energy of the Cooper pairs. This effect of the inertia of the Cooper pairs contributes what is known as the kinetic inductance.
At finite temperatures below the superconducting transition temperature $T_{c}$, a small fraction of the electrons are thermally excited out of the Cooper pair states. These "quasiparticles" produce the small surface resistance $R_{S}$. The net result is that the superconductor has a surface impedance to ac currents $Z_{s}=R_{s}+i \omega L_{s}$ that is almost purely inductive, $\omega L_{S} \gg R_{S}$. The Cooper pairs are bound together by the electron-phonon interaction with an energy $2 \Delta \approx 3.5 k T_{c}$. Photons with energies $>2 \Delta$ that are absorbed by the superconductor can break apart Cooper pairs, resulting in an increase in the quasiparticle density and therefore a change in the surface impedance $Z_{s}$. By measuring this change in the impedance, which alters both the resonant frequency and the resonator quality factor, the optical load on the superconductor can be derived. For aluminum, the binding energy corresponds to a minimum photon frequency of $90 \mathrm{GHz}$.

To implement this as a photon detector, we use thin superconducting films of aluminum and niobium as part of a lithographed resonator circuit (Figure 1). The hybrid quarter-wave resonators are coupled via microstrip to lithographic planar multi-slot antennas, and are also capacitively coupled to a coplanar-waveguide feedline for the microwave readout. Each pixel consists of an antenna, an in-phase combining microstrip feed network, on-chip filters for bandpass definition, and four res- 


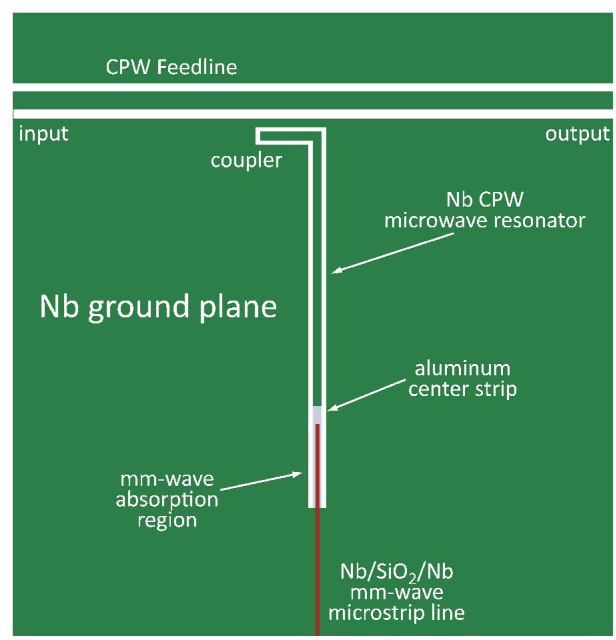

FIGURE 1. A microstrip-coupled quarter-wave $\mathrm{Al} / \mathrm{Nb}$ resonator. Photons with $v>90 \mathrm{GHz}$ are absorbed in the aluminum center strip. A microwave readout signal excites the resonator via the coupler section; the length of the coupler and its distance from the feedline determine $Q_{c}$, the coupling $Q$.

onators (Figure 2). The MKID camera will have 16 tiles of $6 \times 6$ spatial pixels in four colors $(230,290,350,405$ $\mathrm{GHz}=1.3 \mathrm{~mm}, 1.1 \mathrm{~mm}, 850 \mu \mathrm{m}, 750 \mu \mathrm{m}$, matched to the atmospheric transmission windows) for a total of 2304 channels (144 MKIDs/tile $\times 16$ tiles). The simultaneous multicolor imaging will both provide spectral information on sources and aid in sky noise removal.

Away from resonance, the transmission of each quarter-wave resonator is unity. The quality factors are very high, $>10^{4}$, so large numbers of resonators $\left(\sim 10^{2}-10^{3}\right)$ can be multiplexed on a single feedline.

MKIDs have a number of advantages for astronomical instrumentation. The detector fabrication is simple, requiring only $\sim 6$ levels of lithography. Since generationrecombination noise is exponentially suppressed at temperatures well below the transition temperature, where the MKIDs are operated, $T_{c}$ uniformity is not an issue. Modest variations in thickness are also allowable. Finally, because of the multiplexibility of the readout, the focal plane is drastically simplified. Each MKID tile requires only a single cryogenic HEMT amplifier and a pair of coaxial cables for the readout; all of the complicated electronics (for demodulation and decimation) are moved to room temperature.

Furthermore, in our implementation both the multislot antennas that are used to photon-couple the MKIDs and the bandpass filters (lumped-element filters comprised of inductors and capacitors) are also lithographed directly on the chip as part of the same fabrication. a)

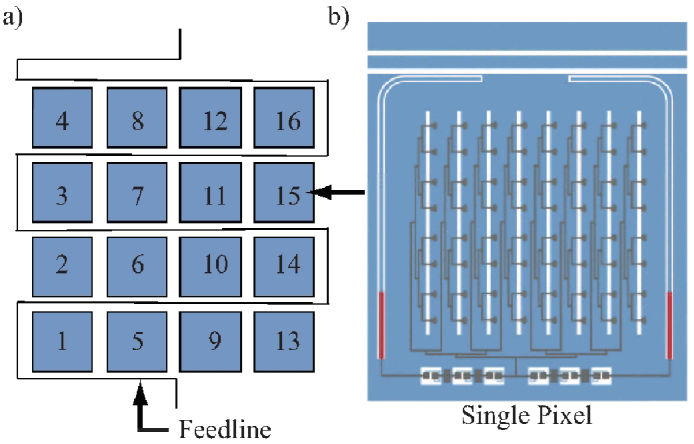

FIGURE 2. An illustration of the $4 \times 4$, two-color pixel array used in the Democam test instrument. (a) shows the layout and the meandered feedline; (b) shows a blow-up of a single pixel, showing the slot antenna, the combining network, the on-chip lumped-element bandpass filters, and the pair of resonators coupled to the feedline.

\section{THE CAMERA}

The design for the camera cryostat was finalized in the Spring of 2008. High Precision Devices Inc. in Boulder, $\mathrm{CO}$ completed fabrication on schedule and delivered the cryostat to JPL in early September of 2008. Cooling to $4 \mathrm{~K}$ is provided by a Cryomech PT-415 pulse-tube cooler; refrigeration of the focal plane to temperatures below the transition temperature of $\mathrm{Al}\left(T_{c}=1.2 \mathrm{~K}\right)$ is accomplished with a Chase Research helium sorption fridge. Figure 3 shows a view of the $4 \mathrm{~K}$ radiation shield and a piece of the $50 \mathrm{~K}$ shield, while Figure 4 shows the $4 \mathrm{~K}$ plate with the mounted Chase fridge.

Thermal and mechanical testing of the cryostat has been carried out at JPL. The fridge performance has been verified under dark conditions. Thermal loading from the UHMWPE window onto the 40K filter stack (and then onto the $4 \mathrm{~K}$ stage) has caused some difficulties; we are implementing thermal filtering, and have already reduced the load on $4 \mathrm{~K}$ to nearly acceptable levels. Accelerometer and thermal stability measurements have revealed that vibration of the pulse-tube cooler is a minor issue. (Thermal fluctuations driven by microphonics will produce fluctuations in the number of thermal quasiparticles in the resonators, and could therefore increase the noise). There are two sources of vibration. One is the external motor. This can be eliminated simply by decoupling the motor from the cryostat, by mounting it to the telescope instead. The second source is the $1.4 \mathrm{~Hz}$ vibration of the pulse tube itself, which is coupled to the $4 \mathrm{~K}$ stage by the copper wick connecting the PT head to the $4 \mathrm{~K}$ plate. This could be suppressed by using a less rigid wick. 


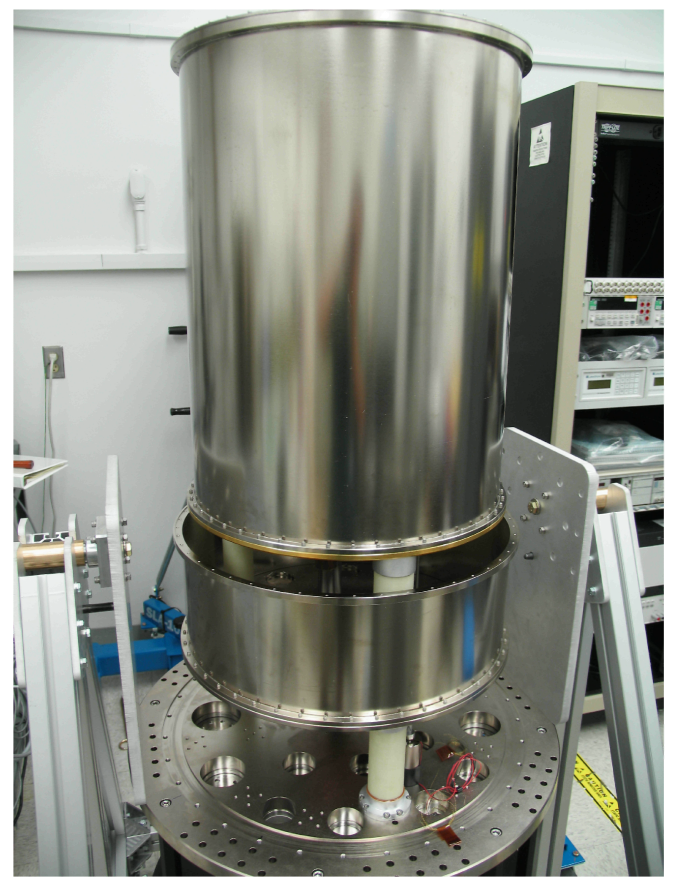

FIGURE 3. Inside the MKID camera cryostat. This shows the $4 \mathrm{~K}$ radiation shield and the split section of the $50 \mathrm{~K}$ shield (at bottom).

TABLE 1. MKID Camera parameters

\begin{tabular}{lrrrr}
\hline No. of spatial pixels: & 576 & & & \\
No. of colors/pixel: & 4 & & & \\
\hline Observing bands & 1 & 2 & 3 & 4 \\
\hline$v_{c e n}(\mathrm{GHz})$ & 230 & 290 & 350 & 405 \\
$\Delta v(\mathrm{GHz})$ & 55 & 45 & 30 & 20 \\
\hline
\end{tabular}

An engineering run at the CSO in Spring 2007 with Democam, a 2-color, 16-pixel prototype camera, revealed that MKIDs are extremely sensitive to magnetic fields; merely changing the orientation of the dewar with respect to the Earth's magnetic field would cause resonator frequencies to shift by hundreds of $\mathrm{kHz}$, with arbitrary sign, as a result of varying magnetic field penetration into the superconducting layers. A Cryoperm ${ }^{\mathrm{TM}}$ magnetic shield (refrigerated to $4 \mathrm{~K}$ ) was therefore designed and built and is currently undergoing testing in Democam. Since the MKID response is expected to scale as $|\mathbf{B}|^{2}$, the factor of $\sim 400$ reduction in on-axis field is expected to be sufficient. Preliminary data indicate that the magnetic shield has reduced the $\mathbf{B}$-field-induced resonator frequency shifts by $\sim$ four orders of magnitude, to $20 \mathrm{~Hz} /$ Gauss; this should be compared to a responsivity of $\sim 2 \mathrm{kHz}$ per $\mathrm{K}$ of loading expected at the telescope.

The optical design of the camera is still being finalized. However, it will have a $14^{\prime}$ field of view, nearly twice that of the Bolocam mm-wave bolometer array.

\section{RESONATOR DESIGNS}

The photon energy incident on a MKID resonator can be determined using a microwave probe signal (see following section) to measure the complex transmission amplitude $S_{21}$ (which can be broken into phase and amplitude components) of the readout signal past the resonator. Measurements of the first generation of MKID arrays revealed higher than expected noise; this noise is almost entirely in the phase direction. Subsequent work $[5,6,7,8]$ showed that this excess is two-level system (TLS) noise. TLS noise is believed to result from the presence of tunneling states in amorphous solids, where one or a group of atoms can tunnel between two sites. These tunneling states have electric dipole moments that can couple to the electric fields within the solids. As such, it is sensitive to capacitor geometry in the MKID resonators, since the TLS noise is dominated by the portions of the resonator with the strongest $\mathbf{E}$-fields, namely, the open-circuit, coupler section. The original designs used conventional CPWs. As discussed by [3], an interdigitated capacitor section significantly reduces the TLS noise, and the next generation of arrays, which have been fabricated and are currently being tested, utilize these IDC designs.

The amplitude signal is much less sensitive to TLS noise. To date, amplitude readout has been HEMTnoise limited, but new resonator materials (e.g., TiN) promise to boost the amplitude responsivity well above the HEMT noise.

\section{TESTING}

As detectors, MKIDs have novel features which require the development of new methods for testing and characterizing the resonators. The resonator frequencies and responsivity depend on several parameters, some constant (e.g., the thickness of the superconducting layer), others variable (e.g., $n_{q p}$, the density of quasiparticles in the aluminum portion of the resonator, which depends on the temperature, the optical loading, and the readout power). The techniques devised for measuring quasiparticle lifetimes, resonator $Q \mathrm{~s}$, etc. are discussed by [1].

\section{OPTIMIZATION}

Operation of the MKID detectors as part of an astronomical instrument requires optimization of the detectors for this purpose, and thus a choice of resonator parameters; note also that these choices must be made for the ex- 
pected optical loading, since $Q_{i}$, the internal resonator $Q$, will change as $P_{o p t}$ is varied. Ideally, $Q_{i}=Q_{c}$, the coupling $Q$. Similarly, the readout power used to excite the resonators also alters the number of quasiparticles; the choice $P_{\text {read }} \sim P_{\text {opt }}$ is optimal, with the precise prefactor dependent on the efficiency with which $P_{\text {read }}$ creates quasiparticles . Furthermore, there are trade-offs between maximizing the responsivity of a single detector, and the number of resonators that can be read out in a fixed bandwidth without unacceptable levels of crosstalk. The derivation of the relevant equations for different noise regimes (device noise- or amplifier noise-dominated) and of the optimal $Q$ s and the implications for the camera mapping speed are presented by [4]. The design of the bandpass filters for the four-color pixels has also been optimized to produce minimal overlap power and similar NEFDs when scaled to $1 \mathrm{~mm}$ in all bands.

\section{READOUT}

MKIDs are read out using homodyne mixing to measure the time-dependent $S_{21}$ of the resonator. Since, as noted above, the off-resonance transmission of the MKIDs is $\simeq 1$, the readout of a large number of resonators can be multiplexed using a single probe signal that contains a comb of frequency tones matched to each MKID. The multiplexed probe signal will be generated at baseband frequency $\left(v_{b} \simeq 0-200 \mathrm{MHz}\right.$ ) using high-speed, largebandwidth DACs to play back a predefined, easily modified waveform stored in memory. Easy modification of the waveform is required because the resonant frequencies of the MKIDs change as a function of observing conditions (i.e., telescope elevation angle or amount of water vapor). The baseband DAC signals are then upconverted to the microwave band and sent into the cryostat to excite the resonators. By using a dual-DAC scheme with an up-converting IQ mixer, and a similar scheme with dual ADCs on the output side, the need for single-sideband conversion is avoided.

Each 144-resonator tile will have its own readout module. The number of resonators per tile is driven by bandwidth constraints: at present, high-speed ADCs with sufficient bit-depth are limited to a few hundred $\mathrm{MHz}$ bandwidth. (This constraint could be circumvented with carrier suppression, but we have chosen to avoid this complexity.) With operating (resonant) frequencies of a few GHz, quality factors $Q \sim 10^{4}$, and a priori uncertainties of a few $\mathrm{MHz}$ in the resonator frequencies, the nominal spacing between resonators must be $\sim 3 \mathrm{MHz}$ to avoid collisions (substantial response overlap) between more than a small fraction of resonators. Since the cutting edge for 14-bit ADCs and DACs is advancing rapidly, the number of resonators per tile can be expected to increase rapidly over the next few years.
The up-converted digital output must be demodulated to measure the complex transmission amplitudes $S_{21}$ of the readout tones past the resonators, and decimated to a reasonable rate for storage to disk. This will be accomplished by an FPGA core, using an FFT narrowband channelizer. Each readout module (one per tile) is being built around a Xilinx SX95T FPGA. The readout electronics, including both the digital and the analog (IF) systems, are being developed and built by Omnisys Instruments $\mathrm{AB}$.

\section{SUMMARY}

Development of the MKID camera is proceeding toward deployment at the CSO in mid-2010. We are confident, based on measured lab optical efficiency and resonator responsivity, and the TLS noise reduction achieved with the new IDC resonator designs, that we will be able to obtain near-BLIP performance for the submillimeter bands at Mauna Kea.

\section{ACKNOWLEDGMENTS}

The MKID Camera project is supported by NSF grant AST-0705157 to the University of Colorado, NASA grant NNGC06C71G to Caltech, the Gordon and Betty Moore Foundation, and the JPL Research and Technology Development Fund. We are grateful to the Xilinx corporation for their generous donation of the FPGAs needed for the readout electronics.

\section{REFERENCES}

1. N.G.. Czakon et al., Microwave kinetic inductance detector (MKID) camera testing for submillimeter astronomy, this conference, (2009).

2. B. Mazin, Microwave Kinetic Inductance Detectors, this conference, (2009).

3. O. Noroozian, Two-Level-System noise reduction for Microwave Kinetic Inductance Detector, this conference, (2009).

4. J. Schlaerth, MKID Camera Submillimeter and Millimeterwave sensitivities, this conference, (2009).

5. J. Gao, A. Vayonakis, S. Kumar, J. Zmuidzinas, M. Daal, B. Sadoulet, B. Mazin, P. K. Day, and H. G. Leduc, Appl. Phys. Letters, 92, 152505, (2008).

6. Gao, J., Zmuidzinas, J., Mazin, B.A., LeDuc, H.G., and Day, P.K., Appl. Phys. Letters, 90, 102507, (2008).

7. J. Gao, M. Daal, J. M. Martinis, B. A. Mazin, P. K. Day, H. G. Leduc, A. Vayonakis, B. Sadoulet, and J. Zmuidzinas, Appl. Phys. Letters, 92, 212504, (2008).

8. S. Kumar, J. Gao, J. Zmuidzinas, B. A. Mazin, H. G. LeDuc, and P. K. Day, Appl. Phys. Letters, 92, 123503, (2008). 